\title{
Worksharing, pricing and competition in the postal sector ${ }^{1}$
}

\author{
Etienne Billette de Villemeur \\ University of Toulouse (IDEI and GREMAQ) \\ 21 Allée de Brienne \\ 31000 Toulouse \\ Helmuth Cremer, ${ }^{2}$ \\ University of Toulouse (IDEI and GREMAQ) \\ 21 Allée de Brienne \\ 31000 Toulouse \\ Bernard Roy and Joëlle Toledano ${ }^{3}$ \\ La Poste \\ 44 Bvd Vaugirard \\ 75757 Paris Cedex 15
}

July 2004

\footnotetext{
${ }^{1}$ We would like to thank Michael Crew, Philippe De Donder and Paul Kleindorfer for their comments.

${ }^{2}$ Corresponding author; e-mail: helmut@cict.fr

${ }^{3}$ The views expressed in this paper are those of the authors and do not necessarily reflect the views of La Poste.
} 


\section{Introduction}

In the postal sector, the practice of worksharing has been introducing a measure of competition even when the industry was otherwise monopolistic. When the customers have the possibility to "bypass" part of the postal network, there is effectively competition in the relevant segments between the operators activities and those of the customers. From that perspective, one can think of the processing of workshared mail as a form of "downstream access" which is provided to the customers. The relevant question is then to know how the workshared product ought to be priced and more generally, how the possibility of worksharing ought to affect the operators pricing structure. This subject has been extensively studied in the literature see Billette de Villemeur et al. (2002, 2003a). ${ }^{1}$

When the market is liberalized and when entry occurs, a new kind of demand for workshared mail may emerge if the other operators do not have their own delivery network, at least in some areas. The pricing of the services provided to competing operator raises the problem of "access pricing". The phenomenon of "downstream access" has been heavily debated in many network industries like telecommunication, electricity and gas, as part of the ongoing liberalization process; see for instance Laffont and Tirole $(1996,2000)$ and Armstrong (2002). A few recent papers have also looked at this problem for the postal sector, see Crew and Kleindorfer (2002) and Billette de Villemeur et al. (2003b,c). However, these contributions neglect the more traditional form of worksharing by customers. A fully fledged model of postal sector pricing would have to account for both the customers and the competitors demand for workshared mail. Panzar (2003) represents a first step in that direction. He uses the worksharing model of Billette de Villemeur et al. (2003a) in which he introduced upstream and downstream competition. However, he uses a rather specific setting and he does not characterize the optimal pricing structure. The current paper tries to bring together competitive access and monopoly worksharing models in a more systematic way.

Before proceeding it may be interesting to take a look at the fundamental issues

\footnotetext{
${ }^{1}$ See also Crew and Kleindorfer (1995), Mitchel (1999), Sherman (2001) and Panzar (2002).
} 
underlying our problem. The regulatory design of postal prices including those for workshared mail is essentially a Ramsey-Boiteux pricing problem. The underlying issues are very simple. The incumbent operator offers different products to different types of customers and we can think of workshared mail as one of these products. While some of these products are final goods, some like workshared mail may be intermediate goods which are used as inputs by other firms. The pricing of this intermediate good then indirectly determines the prices paid by the final consumers of these products. In a "perfect" (first-best) world the pricing rules for all these products are very simple, namely, (long-run) marginal cost for all products. This provides consumers with the correct signals and ensures that the decentralized outcome is efficient.

In an industry like the postal sector, where technology involves "fixed" costs (like the cost of maintaining the delivery network) it is however, typically the case that marginal cost (even long-run marginal costs) are well below average costs. ${ }^{2}$ Strict marginal cost pricing is then problematic because it implies that the operator cannot break even, which is usually considered as not acceptable for a number of reasons (including political economy considerations). ${ }^{3}$ Consequently, one would have to impose positive markups on at least some products in order to meet the break-even constraint. The determination of these markups is precisely what the Ramsey-Boiteux problem is all about. The question is simply how to distort the different prices away from marginal cost in order to breakeven while keeping the efficiency cost of these distortions as small as possible. The exact specification of this problem and hence the specific results depend on the characteristics of the industry (costs, technology and demand) the general regulatory environment (e.g., the presence of a uniform pricing constraint) and on the type of competition there is between the incumbent and the entrant(s) (competitive fringe, monopolistic competition, some form of oligopoly, etc.). The literature so far has concentrated on the competitive fringe case for which a number of interesting results have been obtained. ${ }^{4}$

\footnotetext{
${ }^{2}$ Like most of the regulation literature we use the term fixed cost for the part of cost which is independant of output, even in the long run.

${ }^{3}$ In a first-best setting this problem can be overcome by a lump-sum tranfer to the operator covering its fixed cost.

${ }^{4}$ See e.g., Cremer et al. (1995, 1997), Crew and Kleindorfer (2002), De Donder et al. (2002, 2003), Billette de Villemeur et al. (2003b).
} 
Pricing rules for final goods and for workshared mail are typically inverse-elasticity rules, properly amended to account for cross-price effects (if any). Consequently, optimal prices depend on demand consideration and not just on cost considerations. This is different from the first best setting where prices simply reflect marginal costs. In a recent contribution, Billette de Villemeur et al. (2003c) show that these rules can be generalized to account for imperfect competition

Summing up, it appears that the economists' toolbox regarding pricing in the postal sector, though still in need to be expanded, does already have the potential to offer valuable guidance in the regulatory debate. In many instances, however, all these studies are ignored by the various parties involved, regulators and postal representatives alike. Instead, the debate concentrates on the relative merits of two essentially ad hoc rules (or classes of rules). The first of these ad hoc approaches is the so called Efficient Component Pricing Rule (ECPR) which (roughly speaking) consists in applying the same (per unit) markup on workshared that is applied on the corresponding final product offered by the incumbent operator. Consequently, worksharing per se does not appear to affect the incumbent's profits and more generally its ability to cover its fixed costs. This rule has the theoretical merit that it leads (under some conditions) to efficient entry decisions: entry occurs if and only if the entrant is more efficient. However, these apparent properties often do not stand under closer scrutiny because ECPR does not account for the demand side of the problem. Further, the rule is incomplete and does not explain how the markup on the incumbent's relevant final product ought to be determined. In addition and most significantly, it does not in general yield an efficient Ramsey-Boiteux pricing, even when one ignores the determination of all the other prices.

Another ad hoc rule which is sometimes advocated by regulators (for instance in the UK) is a "cost-plus" rule, where the access price is obtained from the long run marginal cost by applying some ad hoc markup. This may at first sound similar to a Ramsey-Boiteux approach and there exists of course a specific level of the markup for which we obtain the Ramsey price (or alternatively the ECPR level). The crucial difference, however, is that no effort in made to optimize the markup by accounting for instance for demand considerations. Instead the markup is set in a ad hoc way. For 
instance a small markup is added in order to cover the cost of universal service which in turn is calculated in a questionable way (and thus often significantly underestimated; see Cremer et al. (2000)). There is no reason to expect that such a procedure can yield anything which comes close to the optimal prices.

The main features of our setting are as follows. We consider a stylized representation of the postal sector, with two activities (e.g. distribution and a composite activity) and two types of operators: the incumbent universal service operator on the one hand and the potential entrants on the other hand. There are two types of customers and at least three different products. Households consume single piece mail which uses the entire network. Business customers may or may not engage in worksharing depending on the price structure. Business customers can also consume the entrant's product. The incumbent operator processes workshared mail which emanated both from its customers and from the competitors. The incumbent's and the entrants' product may or may not be perfect substitutes.

We start by characterizing the first-best allocation which essentially involves marginal cost pricing. Consequently, differences in prices reflect solely differences in marginal costs. Then we proceed to a more realistic setting where a fixed network cost has to be covered through the pricing scheme.

Our model can be used to study a number of settings depending on the specific assumptions which are made on demand, costs and on the regulatory environment. The current paper is meant to be a first step and we concentrate on a very stylized setting. Specifically, we assume throughout that the entrant does not deliver (there is no bypass). We also concentrate on the case where the access price for competitors is restricted to equal the rate charged for workshared mail to customers. We make no specific assumption at first on the degree of product differentiation between the incumbents' and entrant's product. However, we also look at the special case of perfect substitutes for which crisper results emerge. 


\section{Model}

\subsection{Operators, customers and preferences}

There are two operators: the incumbent universal service operator $I$ and an entrant $E$. The stylized postal network we consider consists of two segments. Segment 1 corresponds to a composite activity including collecting, sorting and transportation. This activity implies a constant marginal cost of $c_{1}$ for operator $I$ and of $k_{E}$ for operator $E$. Segment 2 is delivery. Operator $I$ 's marginal delivery cost is $c_{2}$; Operator $E$ does not have its own delivery network. In addition, there is a fixed cost of $F^{I}>0$ for operator $I$ and $F^{E} \geq 0$ for operator $E$.

There are two types of senders and two goods. The $n_{h}$ senders of type $h$ (households) consume good $x$ which is supplied by $I$ and uses both segments. The marginal cost of $x$ is thus given by $c_{1}+c_{2}$. The $n_{f}$ customers of type $f$ may or may not use segment 1 of operator I's network. If they do not use segment 1 they consume good $z$ which implies a marginal cost of $k+c_{2}$, where $k$ is distributed over $[\underline{k}, \bar{k}]$ according to the cumulative distribution $G(k)$ with density $g(k)$. Observe that $c_{2}$ is the operator's cost, while $k$ is directly born by the customer. Alternatively, they can consume good $x$ for which they pay the same price as households, ${ }^{5}$ or good $y$ supplied by operator $E$. Prices are given by $p_{x}$ and $p_{z}$ for operator $I$ and by $p_{y}$ for operator $E$.

Let $S_{h}\left(x_{h}\right)$ denote the (gross) surplus of a households, while $S_{f}\left(x_{f}+z, y, k\right)$ represents the (gross) surplus of a sender of type $f$ with characteristic $k{ }^{6}$ Observe that $k$ represents not only the preparation cost, but also determines surplus and hence demand. Net surplus is obtained by subtracting total cost: payment to the operator plus cost of activity 1 , if applicable. The demand function for a household is given by:

$$
x_{h}(p)=\arg \max _{x}\left\{S_{h}(x)-p x\right\} .
$$

\footnotetext{
${ }^{5}$ Except for the cost difference $x$ and $z$ are considered as perfect substitutes.

${ }^{6}$ For simplicity we use surplus as a welfare measure for firms. From a strict welfare economics point of view, this can be understood as representing the surplus of the consumers who buy the goods produced by firms $f$ which use postal services as inputs. One can easily show that our shortcut does not involve any loss of generality in the case where all downstream markets are competitive.
} 
The demand functions of the representative sender $f$ are obtained by maximizing

$$
\begin{aligned}
& S_{f}\left(x_{f}+z, y, k\right)-p_{x} x_{f}-\left(p_{z}+k\right) z-p_{y} y \\
& \text { s.t } \quad x_{f}, z \geqslant 0 .
\end{aligned}
$$

To characterize the solution to this problem define $\widetilde{k}=p_{x}-p_{z}$. This yields the demand functions:

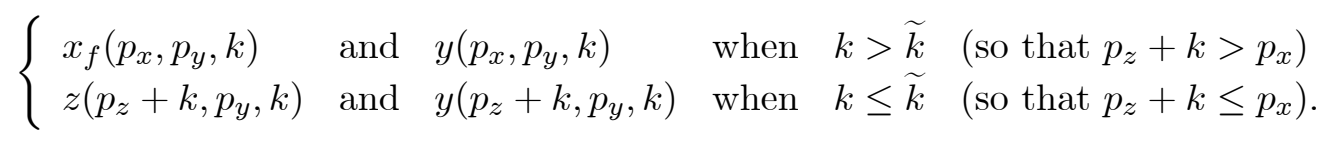

To understand the determination of the demand functions, note that all users of type $f$ for which $p_{z}+k \leq p_{x}$ (i.e., when $k \leq p_{x}-p_{z}=\widetilde{k}$ ) find it profitable to buy good $z$ (rather than $x$ ) at a level $z\left(p_{z}+k, p_{y}, k\right)$. Overall per-unit cost of $z$ is equal to $p_{z}+k$; it is this overall cost rather than just $p_{z}$ which determines demand. On the other hand, when $p_{z}+k>p_{x}$, it is cheaper to consume $x$ (which is otherwise a perfect substitute of $z$ ) and demand is $x_{f}\left(p_{x}, p_{y}, k\right)$. Observe that when $p_{z}+k=p_{x}$ we have $x_{f}\left(p_{x}, p_{y}, k\right)=z\left(p_{z}+k, p_{y}, k\right)$; this property arises because, except of cost considerations, $x$ and $z$ are perfect substitutes. Either way, the firms may also consume the (differentiated) product offered by $E$, at levels specified by $y(\cdot)$.

Substituting demand functions into net surplus yields the following indirect utility functions:

$$
\begin{aligned}
& V_{h}\left(p_{x}\right)=S_{h}\left[x_{h}\left(p_{x}\right)\right]-p_{x} x_{h}\left(p_{x}\right), \\
& V_{f}\left(p_{x}, p_{z}, p_{y}, k\right)=\left\{\begin{array}{l}
S_{f}\left[z\left(p_{z}+k, p_{y}, k\right), y\left(p_{z}+k, p_{y}, k\right), k\right] \\
-\left(p_{z}+k\right) z\left(p_{z}+k, p_{y}, k\right)-p_{y} y\left(p_{z}+k, p_{y}, k\right) \\
\text { if } p_{z}+k \leq p_{x}, \\
S_{f}\left[x_{f}\left(p_{x}, p_{y}, k\right), y\left(p_{z}+k, p_{y}, k\right)\right] \\
-p_{x} x_{f}\left(p_{x}, p_{y}, k\right)-p_{y} y\left(p_{z}+k, p_{y}, k\right) \\
\text { if } p_{z}+k>p_{x} .
\end{array}\right.
\end{aligned}
$$

\subsection{Market demand, cost and profits}

We are now in a position to determine market demand functions for the various (final) 
goods. The aggregate demand functions for the incumbent products are:

$$
\begin{aligned}
& X\left(p_{x}, p_{z}, p_{y}\right)=n_{h} x_{h}\left(p_{x}\right)+n_{f} \int_{p_{x}-p_{z}}^{\bar{k}} x_{f}\left(p_{x}, p_{y}, k\right) g(k) d k \\
& Z\left(p_{x}, p_{z}, p_{y}\right)=n_{f} \int_{\underline{k}}^{p_{x}-p_{z}} z\left(p_{z}+k, p_{y}, k\right) g(k) d k
\end{aligned}
$$

The demand function for the operator $E$ is:

$$
\begin{aligned}
Y\left(p_{x}, p_{z}, p_{y}\right)= & n_{f} \int_{\underline{k}}^{p_{x}-p_{z}} y\left(p_{y}, p_{z}+k, k\right) g(k) d k \\
& +n_{f} \int_{p_{x}-p_{z}}^{\bar{k}} y\left(p_{y}, p_{x}, k\right) g(k) d k
\end{aligned}
$$

Expression (3) provides the total demand for the incumbent's single piece mail. The first term on the RHS of this expression is the total demand by households, while the second term represents the total demand by the firms who do not workshare their mail (those with high levels of $k$ ). Expression (4) gives the firms' total demand for commercial (workshared) mail. It is the sum of individual demand levels for the firm with a low level of $k$. Finally, equation (5) represents the demand level for the entrants product. This demand potentially emanates from all firms; however, we do not rule out the case where $y=0$ for some firms and especially those with a low level of $k$. This will of course be particularly relevant when the products are close substitutes; see the case of perfect substitutes below.

For the remainder of the paper, it is important to keep in mind that these demand levels are for final products (as opposed to intermediate goods). Since operator $E$ does not deliver, $Y$ will also be delivered through the incumbent's network. Consequently, the total (final and intermediate) demand for the incumbent's workshared mail will effectively be equal to $Z+Y$.

Per the assumptions introduced in subsection 2.1 we can write the costs functions as follows.

$$
\begin{aligned}
C^{I} & =\left(c_{1}+c_{2}\right) X+c_{2}(Z+Y)+F^{I} \\
C^{E} & =\left(k^{E}+p_{z}\right) Y+F^{E}
\end{aligned}
$$


To understand these expressions, recall that marginal costs are constant, that $X$ used both segments of the network, while the $(Z+Y)$ units workshared mail only use the delivery network. Then entrant pays $p_{z}$ in lieu of the delivery cost and it has a preparation cost of $k^{E}$ (rather than $c_{1}$ for the incumbent).

Finally, profits can be expressed as follows.

$$
\begin{aligned}
\pi^{I} & =p_{x} X+p_{z}(Z+Y)-C^{I}, \\
& =\left[p_{x}-\left(c_{1}+c_{2}\right)\right] X+\left[p_{z}-c_{2}\right](Z+Y)-F^{I}, \\
\pi^{E} & =p_{y} Y-C^{E} \\
& =\left[p_{y}-\left(k^{E}+p_{z}\right)\right] Y-F^{E} .
\end{aligned}
$$

Observe that both costs and profits can be expressed as functions of prices (and of the exogenous cost parameters). To obtain these functions is is sufficient to substitute aggregate demand functions (3)-(5) in the expressions for costs and profits (6)-(9).

Our formal analysis concentrates on the competitive fringe case, where $E$ price at marginal cost so that $p_{y}=k^{E}+p_{z}$. This assumption is of course only meaningful if we assume $F^{E}=0$.

\section{First-best solution}

The first-best solution constitutes an interesting benchmark. To obtain it, we maximize total surplus which is given by

$$
W=V_{h}\left(p_{x}\right)+\int_{\underline{k}}^{\bar{k}} V_{f}\left(p_{x}, p_{z}, p_{y}, k\right)+\pi^{I}\left(p_{x}, p_{z}, p_{y}\right)+\pi^{E}\left(p_{x}, p_{z}, p_{y}\right),
$$

with respect to prices $\left(p_{x}, p_{z}, p_{y}\right)$. The result is well known and we skip the derivation here. ${ }^{7}$ Differentiating (10) with respect to $\left(p_{x}, p_{z}, p_{y}\right)$ and rearranging yields the following solution:

$$
\begin{aligned}
& p_{x}=c_{1}+c_{2}, \\
& p_{z}=c_{2}, \\
& p_{y}=k^{E}+c_{2} .
\end{aligned}
$$

\footnotetext{
${ }^{7}$ It can be obtained from expressions (27)-(28) in the Appendix by setting $\lambda=0$.
} 
In words, all prices are set at marginal cost. Event though these expressions are standard, it has to be pointed out that they have an number of interesting implications in our setting.

First, we can think about $p_{x}-p_{z}$ as the "worksharing discount". From (11) and (12) it follows immediately that this discount is simply equal to $c_{1}$, that is the avoided cost. This in turn implies that we have "production efficiency" in the sense that a firm will workshare its mail if and only if its preparation cost is less than that of the operator. To see this recall that all firms with $k<\widetilde{k}=p_{x}-p_{z}$ consume workshared mail and under marginal cost pricing this reduces to $k<c_{2}$.

Second, we have assumed from the outset that $p_{z}$ represents both the discounted price charged to customers and the access price charged to the competitor. Put differently, price discrimination between competitors and customers has been ruled out. It is interesting to note that in a first-best setting this does not effectively impose a restriction. Since the (marginal) delivery cost is the same whatever the "origin" of the mail product, its price ought to be the same for everyone anyway.

Third, we have solved this problem as if the regulator would control directly the entrant's price $p_{y}$. This is a technical simplification which is of no relevance in the competitive fringe case where $p_{y}=k^{E}+p_{z}$ so that when $p_{z}$ is set according to (12), (13) is automatically satisfied. Finally, one can easily verify that with first-best prices, operator $I$ realizes a deficit equal to $F^{I}$. Put differently, revenues do not cover fixed costs and the first-best solution is not feasible when the operator is required to break even. We now turn to the second best problem which arises when budget balancing is imposed.

\section{Second-best solution}

Set $p_{y}=k^{E}+p_{z}$ (competitive fringe) and maximize the welfare function subject to the constraint that the incumbent realizes nonnegative profits. The Lagrangian is given by:

$$
L=V_{h}\left(p_{x}\right)+\int_{\underline{k}}^{\bar{k}} V_{f}\left(p_{x}, p_{z}, p_{y}, k\right)+(1+\lambda) \pi^{I}\left(p_{x}, p_{z}, p_{y}\right)+\pi^{E}
$$


where $\lambda>0$ is the Lagrange multiplier of the firms break-even constraint. Observe that $\pi^{E} \equiv 0$ by assumption (competitive fringe and $F^{E}=0$ ). The FOCs are as follows:

$$
\begin{aligned}
\frac{\partial L}{\partial p_{x}}= & -x_{h}\left(p_{x}\right)-\int_{p_{x}-p_{z}}^{\bar{k}} x_{f}\left(p_{x}, p_{y}, k\right) g(k) d k \\
& +(1+\lambda)\left[X+\left[p_{x}-\left(c_{1}+c_{2}\right)\right] \frac{\partial X}{\partial p_{x}}+\left[p_{z}-c_{2}\right]\left(\frac{\partial Z}{\partial p_{x}}+\frac{\partial Y}{\partial p_{x}}\right)\right], \\
\frac{\partial L}{\partial p_{z}}= & -\int_{\underline{k}}^{p_{x}-p_{z}} z\left(p_{z}+k, p_{y}, k\right) g(k) d k \\
& -\frac{d p_{y}}{d p_{z}}\left[\int_{\underline{k}}^{p_{x}-p_{z}} y\left(p_{z}+k, p_{y}, k\right) g(k) d k+\int_{p_{x}-p_{z}}^{\bar{k}} y\left(p_{x}, p_{y}, k\right) g(k) d k\right] \\
& +(1+\lambda)\left[Z+Y+\left[p_{x}-\left(c_{1}+c_{2}\right)\right]\left(\frac{\partial X}{\partial p_{z}}+\frac{\partial X}{\partial p_{y}} \frac{d p_{y}}{d p_{z}}\right)\right] \\
& +(1+\lambda)\left[\left[p_{z}-c_{2}\right]\left(\frac{\partial Z}{\partial p_{z}}+\frac{\partial Y}{\partial p_{z}}+\frac{\partial Z}{\partial p_{y}} \frac{d p_{y}}{d p_{z}}+\frac{\partial Y}{\partial p_{y}} \frac{d p_{y}}{d p_{z}}\right)\right],
\end{aligned}
$$

where $d p_{y} / d p_{z}=1$. For the sake of interpretation it is interesting to redefine the demand functions to account for the induced price variation of the entrant:

$$
\begin{aligned}
\widetilde{X}\left(p_{x}, p_{z}\right) & =X\left(p_{x}, p_{z}, p_{y}\left(p_{z}\right)\right), \\
\widetilde{Y}\left(p_{x}, p_{z}\right) & =Y\left(p_{x}, p_{z}, p_{y}\left(p_{z}\right)\right), \\
\widetilde{Z}\left(p_{x}, p_{z}\right) & =Z\left(p_{x}, p_{z}, p_{y}\left(p_{z}\right)\right),
\end{aligned}
$$

where $p_{y}\left(p_{z}\right)=k^{E}+p_{z}$. Observe that these demand functions $\widetilde{X}, \widetilde{Y}$ and $\widetilde{Z}$ only depend on operator I's prices which is convenient because these prices are the relevant decision variables in our problem.

We show in the appendix that the FOC can be rearranged to yield the following pricing rules:

$$
\begin{aligned}
\frac{p_{x}-\left(c_{1}+c_{2}\right)}{p_{x}} & =\frac{\lambda}{1+\lambda} \frac{1}{\widehat{\sigma}_{X}} \\
\frac{p_{z}-c_{2}}{p_{z}} & =\frac{\lambda}{1+\lambda} \frac{1}{\widehat{\sigma}_{Y+Z}}
\end{aligned}
$$

where $\widehat{\sigma}_{X}$ and $\widehat{\sigma}_{Y+Z}$ are the superelasticities of $\widetilde{X}$ and $\widetilde{Y}+\widetilde{Z}$ respectively. These expressions are the counterparts to equations (15) and (16) obtained by Billette de Villemeur et al. (2003a) for the monopoly case. To interpret them, one has to recall 
first that superelasticities are generalized demand elasticities which account for crossprice effects. When demand functions are independent, superelasticities are equal to ordinary price elasticities. In that case (19) and (20) reduce to "inverse elasticity rules". However, in our setting demand functions are not independent. A variation in one of the prices affects demand levels of the other goods and this brings about distortions which are accounted for in the superelasticities. Observe that when the goods are substitutes (as is the case here) one can show that superelasticities are smaller than ordinary price elasticities.

In spite of this complication it remains that the pricing rules have the familiar flavor and interpretation of Ramsey type rules. This point was made by Billette de Villemeur et al. (2003a) for a monopoly setting. Here we obtain similar expressions under competition. The main difference is that we have $Y+Z$ rather than just $Z$ in the second expression. In either case this has to be interpreted as the total demand for workshared mail which here also emanates from the competing operators. The interesting feature is that while the incumbent's and the entrant's products are not perfect substitutes (for the customers) they are here treated as if they were perfect substitutes: only the sum of demands matters. This is because from operator $I^{\prime} s$ perspective the two types of mail are indistinguishable; they have the same marginal cost (delivery only) and they are priced alike.

Combining these pricing expression, we can determined the second best worksharing discount which is given by:

$$
p_{x}-p_{z}=c_{1}+\frac{\lambda}{1+\lambda}\left(\frac{p_{x}}{\widehat{\sigma}_{X}}-\frac{p_{z}}{\widehat{\sigma}_{Y+Z}}\right) .
$$

Under the plausible assumption that $\widehat{\sigma}_{Y+Z}>\widehat{\sigma}_{X}$ expression (21) implies that $p_{x}-p_{z}>$ $c_{1}$. Observe that the application of the ECPR rule would require the discount to equal avoided costs, namely $c_{1}$. This would amount to apply the tradeoff which warrants firstbest production efficiency. Not surprisingly this rule has to be amended in a secondbest setting and under plausible assumption on the demand elasticities one can expect the discount to exceed avoided cost. Comparing this expression to its counterpart in Billette de Villemeur et al. (2003a), namely equation (17) it appears that the presence 
of competition does not appear to fundamentally affect the structure of the pricing rule. In either case, we obtain a rule which can be qualified as "ECPR, adjusted for demand (super)elasticities". This does of course not imply that the actual levels prices are the same or are similar. The demand functions underlying the superelasticities are affected by the presence of competition. We shall have a closer look at this issue for the special case of perfect substitutes to which we now turn.

\section{The case of perfect substitutes}

We now turn to the special case where the incumbent and the entrant's products are perfect substitutes. Observe that this is the case on which Panzar (2003) concentrates. Though extreme, this does not appear to be a totally meaningless setting to consider here. One can argue that any degree of differentiation there may be between the two operators is likely to be mitigated by the fact that operator $I$ uses operator $E$ 's delivery network. In other words, once they get to the delivery segment the three products $X$, $Y$ and $Z$ become indistinguishable.

With $p_{y}=k^{E}+p_{z}$ (competitive fringe), the demand of type $f$ customers has the following properties:

$$
\left\{\begin{array}{llll}
x_{f}=0 ; & y=0 ; & z_{k}>0 & \text { if } k<\inf \left\{k^{E}, p_{x}-p_{z}\right\} \\
x_{f}=0 ; & y>0 & z_{k}=0 ; & \text { if } k^{E}<\inf \left\{k, p_{x}-p_{z}\right\} \\
x_{f}>0 & y=0 ; & z_{k}=0 ; & \text { if } p_{x}-p_{z}<k^{E}<k
\end{array}\right.
$$

Observe that demand function are now discontinues when $k^{E}<p_{x}-p_{z}=\widetilde{k}$. To determine the solution, two regimes have to be considered separately. We have either $k^{E} \leq \widetilde{k}$ (Regime $I$ ) or $k^{E}>\widetilde{k}$ (Regime $\left.I I\right)$.

\subsection{Regime $I: k^{E} \leq \widetilde{k}$}

In this case, no customers of type $f$ consume product $x$. The demand functions for the incumbent products are:

$$
\begin{aligned}
X\left(p_{x}\right) & =x_{h}\left(p_{x}\right), \\
Z\left(p_{z}\right) & =\int_{\underline{k}}^{k^{E}} z_{k}\left(p_{z}+k\right) g(k) d k .
\end{aligned}
$$




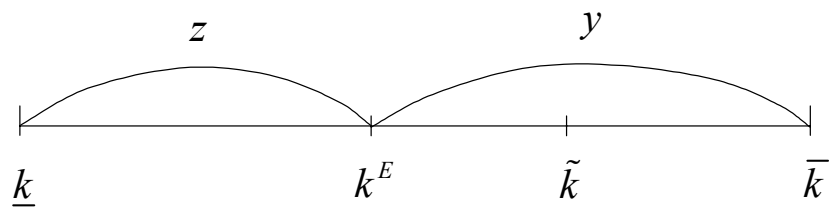

Regime I

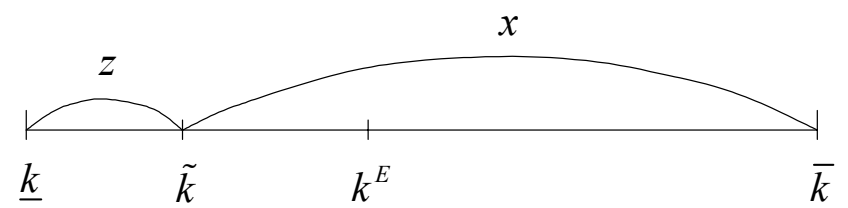

Regime II

Figure 1: Case of perfect substitutes: demand of type $f$ customers in Regimes $I$ and II. 
The demand function for the operator $E$ is:

$$
Y\left(p_{z}\right)=\int_{k^{E}}^{\bar{k}} y_{k}\left(p_{z}+k^{E}\right) g(k) d k
$$

Remark that, since $y$ and $x$ (hence $z$ ) are perfect substitutes, $y_{k}(p)=z_{k}(p)$. As a result, this is "as if" the entry of firm $E$ were allowing firms with $k \geq k^{E}$ to sort their mail at a cost of only $k^{E}$. Firm $I$ is now facing a set of firms with a distribution of $k$ which is cut from above at the level $k^{E}$ and all these firm consume the workshared mail.

Appendix B shows that the optimal pricing rules are now given by

$$
\begin{aligned}
\frac{p_{x}-\left(c_{1}+c_{2}\right)}{p_{x}} & =\frac{\lambda}{1+\lambda} \frac{1}{\varepsilon_{X_{h}}} \\
{\left[\frac{p_{z}-c_{2}}{p_{z}}\right] } & =\frac{\lambda}{(1+\lambda)} \frac{1}{\varepsilon_{Y+Z}}
\end{aligned}
$$

Where $\varepsilon_{X_{h}}$ and $\varepsilon_{Y+Z}$ are ordinary price elasticities (absolute values). Observe that we have returned to ordinary price elasticities rather than superelasticities which where obtained in the general case. This is because, with perfect substitutes, cross-price elasticities (between $X$ on the one hand and $Z+Y$ on the other hand) are zero within a given regime.

\subsection{Regime $I I: k^{E}>\widetilde{k}$}

In the case where $k^{E}>p_{x}-p_{z}$, the demand for the operator $E$ is zero and the demand functions for the incumbent products are:

$$
\begin{aligned}
X\left(p_{x}, p_{z}\right) & =x_{h}\left(p_{x}\right)+\int_{p_{x}-p_{z}}^{\bar{k}} x_{f}\left(p_{x}, k\right) g(k) d k \\
Z\left(p_{x}, p_{z}\right) & =\int_{\underline{k}}^{p_{x}-p_{z}} z\left(p_{z}+k, k\right) g(k) d k
\end{aligned}
$$

and we are back to the model of worksharing. Optimal pricing rules are then given by expressions (15) and (17) in Billette de Villemeur and al. (2003a).

\subsection{Which Regime is relevant?}

The condition defining the applicable regime depends on $\widetilde{k}$ and thus on the prices. Formally, one would have to evaluate welfare at the solutions achieved in either regime 
and compare welfare levels to determine the global optimum. This is a complicated task and the problem cannot be done based on the first order conditions alone. However, it is quite clear that the solution will depend on the relative efficiency of the two operator's on segment 1, i.e. on the comparison between $k^{E}$ and $c_{1}$.

The first interesting property one can show is that when $k^{E} \leq c_{1}$ Regime $I I$ can not occur. In other words, it is never optimal to (totally) exclude an efficient competitor from the market. To see that, recall from Billette de Villemeur et al. (2003a) that Regime $I I$ yields $\widetilde{k}=p_{x}-p_{z}>c_{1}$, hence $k^{E} \leq c_{1}$ is incompatible with the condition for Regime $I I$ namely $k^{E}>\widetilde{k}$.

By continuity, this property implies that when $k^{E}>c_{1}$, but not "too large", Regime $I$ will continue to prevail. In other words, at the second best optimum, it may be interesting to "tolerate" a competitor who is slightly less efficient than the incumbent (even though its product is a perfect substitute to that of operator $I$ ). This is because the presence of $E$ allows for a solution which differentiates between firms with high $k$ (who will consume $Y$ ) and households (who consume $X$ ). If the competitor is not in the market, both of these groups consume $X$ at a uniform price.

Finally, when $k^{E}$ become sufficiently large the solution will imply Regime $I I$; there is no room at a second best optimum for a very inefficient entrant.

\subsection{Discounts under monopoly and under competition}

Having characterized the solution under competition we can now attempt to compare it to the monopoly case. In particular it is interesting to study how the worksharing discount $p_{x}-p_{z}$ is affected by the presence of the competitor. To do this we shall remain within the setting of perfect substitutes.

Not surprisingly, the answer depends (among other things) on the cost of the entrant $k^{E}$. To see this let us start from the monopoly solution denoted $p_{x}^{m}$ and $p_{z}^{m}$, with $\widetilde{k}^{m}=p_{x}^{m}-p_{z}^{m}$. We know from Billette de Villemeur et al. (2003b) that $\widetilde{k}^{m}>c_{1}$ (under plausible assumptions on demand elasticities). Depending on the entrant's cost we can then have three configurations. First, there is the rather trivial case where

the entrant has large preparation costs, $k^{E} \geq \widetilde{k}^{m}$. In that case, we have Regime $I I$ 
and monopoly prices (and discounts) will continue to prevail. Second and at the other extreme, we can have $k^{E}<c_{1}$, which of course implies $k^{E}<\widetilde{k}^{m}$. As shown in the previous subsection Regime $I$ prevails and the solution is given by (22) and (23). To compare these to their monopoly counterparts, expressions (15) and (16) of Billette de Villemeur et al. (2003b), we have to assess the impact of the presence of the competitor on the demand elasticities. Roughly speaking, the firms with the larger $k$ (which are also the ones with the relatively less elastic demand) consume $x$ under monopoly and $y$ (and thus indirectly also $z$ ) under competition. It is quite clear that this tends to make the demand for $X$ less elastic (as the consumers) with the more elastic demand switch to $Y .{ }^{8}$ Consequently, we can expect $p_{x}$ to increase. ${ }^{9}$ Furthermore, the switching consumers have smaller elasticities than the low $k$ firms. Consequently, they will cause the elasticity for $Z$ to be lower than in the monopoly case. But then $p_{z}$ would also tend to increase and the overall impact on $\widetilde{k}$ appears to be ambiguous. ${ }^{10}$

Finally, there is the case where $c_{1} \leq k^{E} \leq \widetilde{k}^{m}$. In words, the entrant's preparation cost is between that of the incumbent and the monopoly level of the worksharing discount. In particular, consider the case where $k^{E}=\widetilde{k}^{m}-\varepsilon$, where $\varepsilon>0$ is small. Now, if operator $I$ maintains its monopoly prices, all consumers to the right of $\widetilde{k}^{m}$ will switch from $x$ to $y$ (while essentially nothing else changes). With $k^{E}>c_{1}$ this switch can only decrease welfare (because total preparation costs increase). This welfare loss can easily be avoided when operator $I$ changes its prices to reduce $\widetilde{k}$ to a level below $k^{E}$. Since $\varepsilon$ is small and since we start from a optimal solution, this variation will have no first order impact on welfare. It does, however, avoid the increase in preparation cost. Consequently, it will clearly dominate the status quo (maintaining monopoly prices upon entry) with higher preparation costs. To sum up, in this case we can conclude unambiguously that competition decreases the level of the discount. Now, as $\varepsilon$ increases, so

\footnotetext{
${ }^{8}$ Tthe demand of firms with high preparation cost is expected to be nevertheless more elastic than that of houselholds.

${ }^{9}$ This is a very rough argument of course because the monopoly expression involve superelasticities. Our arguement assumes thus implicitely that cross-price effects are not too significiant.

${ }^{10}$ All these arguments are for a given level of $\lambda$. In reality $\lambda$ would of course change. Practically speaking this means that when both prices increase (starting from the Ramsey solution) we can expect profits to increase. But then prices can again be lowered without violating the break-even constraint. This introduces yet another effect which of course does not make the result any less ambiguous.
} 
that $k^{E}$ will become closer and closer to $c_{1}$, we can continue to make this argument. However, the price adjustment need to match $k^{E}$ become larger and larger and this will also imply a welfare loss. This has to be balanced against the reduction in preparation cost (which becomes of course smaller and smaller as $k^{E}$ approaches $c_{1}$ ). Thus at some point we will switch to Regime $I$ and return to case where the impact on $\widetilde{k}$ is ambiguous.

\section{Concluding remarks}

The current regulatory debate in the postal sector calls for an in depth study of the appropriate pricing rules in a competitive environment. Such an investigation has to encompass both worksharing discounts granted to customers and the pricing of the mail which is prepared by competitors but delivered by the incumbent postal operator. Building on our earlier paper which had studied the pricing of worksharing under monopoly, we have provided a framework which is suitable to jointly study access and worksharing in a liberalized industry. However, in the current paper we have not fully explored the potential of our framework. We have looked only at one of the possible scenarios and probably one of the simplest. The natural next steps would be for instance to introduce bypass (in delivery), to consider delivery areas with different costs, etc. This is left for future research.

In the meantime we can already learn from our partial results that the optimal prices are not going to be determined according to simple ad hoc rules which moreover would be valid in all circumstances. The pricing rules are complex in the sense that many factors, including demand, cost and regulatory rules will matter. We have already discussed the role of the entrant sorting cost $k^{E}$ in the previous section. More interestingly, one can easily see that the arguments used to asses the impact of entry on the worksharing discount (in Subsection 5.4) crucially depend on the requirement the same $p_{z}$ applies to both to operator $E$ and to customers. The impact of bypass can be expected to be even more significant. As long as there is no bypass operator $I$ set positive markups on all products, including the one sold by competitors to finance its fixed cost. The other operators' products are of course only "taxed" indirectly, through the access charge. However, as long the operator $E$ has no delivery network this is of no relevance. With 
the possibility of bypass, on the other hand, it is more difficult or maybe even impossible to realize markups on the competitor's products. Summing up, the main conclusion to be drawn is thus that the impact of liberalization is very difficult to assess and will very much depend on the exact (competitive) environment which is considered. 


\section{References}

Armstrong, M., (2002), "The theory of access pricing and interconnection", in Handbook of Telecommunication, vol 1., edited by M. Cave et al., 295-386.

Billette de Villemeur, E, H. Cremer, B. Roy. and J. Toledano (2002), "Pricing and worksharing discounts in the postal sector" in Postal and Delivery Services: Delivering on Competition, edited by M. A. Crew and P. R. Kleindorfer, Boston : Kluwer Academic Publishers, 33-48.

Billette de Villemeur, E, H. Cremer, B. Roy. and J. Toledano (2003a), "Optimal pricing and global price-cap in the postal sector", Journal of Regulatory Economics, 24, 49-62.

Billette de Villemeur, E, H. Cremer, B. Roy. and J. Toledano (2003c), "Pricing and imperfect competition in the postal sector", mimeo, IDEI, University of Toulouse.

Billette de Villemeur, E, H. Cremer, B. Roy. and J. Toledano (2004), "Access and (Non-)Uniform Pricing in the Postal Sector" in Competitive Transformation of the Postal and Delivery Sector, edited by M. A. Crew and P. R. Kleindorfer, Boston : Kluwer Academic Publishers, 43-68.

Cremer, H., M. De Rycke and A. Grimaud (1995), "Alternative scenarios for the reform of postal services: optimal pricing and welfare", in Commercialization of Postal and Delivery Services, edited by M. A. Crew and P. R. Kleindorfer, Boston: Kluwer Academic Publishers, 247-267.

Cremer, H., M. De Rycke and A. Grimaud (1997), "Costs and benefits of universal service obligations in the postal sector" in Managing Change in the Postal and Delivery Industries, edited by M. A. Crew and P. R. Kleindorfer, Boston : Kluwer Academic Publishers, 22-41.

Cremer, H., A. Grimaud and J.-J. Laffont (2000), "The cost of universal service in the postal sector" in Current Directions in Postal Reform, 
edited by M. A. Crew and P. R. Kleindorfer, Boston : Kluwer Academic Publishers, 47-68.

Cremer, H., J.P. Florens, A. Grimaud, S. Marcy, B. Roy. and J. Toledano. (2001), "Entry and competition in the postal market : foundations for the construction of entry scenarios", Journal of Regulatory Economics, 19, 107-121.

Crew M. and P. Kleindorfer (1995), "Pricing in postal service under competitive entry", in Commercialization of Postal and Delivery Services, edited by M. A. Crew and P. R. Kleindorfer, Boston: Kluwer Academic Publishers, 117-136.

Crew M. and P. Kleindorfer (2002), "Balancing access and the universal service obligation", in Postal and Delivery Services: Delivering on Competition, edited by M. A. Crew and P. R. Kleindorfer, Boston : Kluwer Academic Publishers, 3-32.

Laffont, J.J. and J. Tirole (1996), "Creating competition through interconnection: Theory and practice", Journal of Regulatory Economics, 10, $227-256$.

Laffont, J.J. and J. Tirole (2000), Competition in Telecommunications, MIT Press, Cambridge MA.

Law, P. (1997), Welfare effects of pricing in anticipation of Laspeyres, Bulletin of Economic research, 49, 17-27.

Panzar, J. (2003), "The economic effects of combining liberalization and unbundling in postal markets", mimeo.

Sherman, R. (2001), "Optimal worksharing discounts", Journal of Regulatory Economics, 19, 81-92. 


\section{A Derivation of expressions (19) and (20)}

The derivatives of the redefined demand functions are given by

$$
\begin{aligned}
& \frac{\partial \widetilde{X}}{\partial p_{x}}=\frac{\partial X}{\partial p_{x}} \text { and } \frac{\partial \widetilde{X}}{\partial p_{z}}=\frac{\partial X}{\partial p_{z}}+\frac{\partial X}{\partial p_{y}} \frac{d p_{y}}{d p_{z}} \\
& \frac{\partial \widetilde{Y}}{\partial p_{x}}=\frac{\partial Y}{\partial p_{x}} \text { and } \quad \frac{\partial \widetilde{Y}}{\partial p_{z}}=\frac{\partial Y}{\partial p_{z}}+\frac{\partial Y}{\partial p_{y}} \frac{d p_{y}}{d p_{z}} \\
& \frac{\partial \widetilde{Z}}{\partial p_{x}}=\frac{\partial Z}{\partial p_{x}} \quad \text { and } \quad \frac{\partial \widetilde{Z}}{\partial p_{z}}=\frac{\partial Z}{\partial p_{z}}+\frac{\partial Z}{\partial p_{y}} \frac{d p_{y}}{d p_{z}}
\end{aligned}
$$

Using (16)-(18) and (24)-(26), the FOCs can be rewritten as:

$$
\begin{aligned}
\frac{\partial L}{\partial p_{x}} & =\lambda X+(1+\lambda)\left[\left[p_{x}-\left(c_{1}+c_{2}\right)\right] \frac{\partial X}{\partial p_{x}}+\left[p_{z}-c_{2}\right]\left(\frac{\partial Z}{\partial p_{x}}+\frac{\partial Y}{\partial p_{x}}\right)\right] \\
\frac{\partial L}{\partial p_{z}} & =\lambda(Z+Y)+(1+\lambda)\left[\left[p_{x}-\left(c_{1}+c_{2}\right)\right] \frac{\partial \widetilde{X}}{\partial p_{z}}+\left[p_{z}-c_{2}\right]\left(\frac{\partial \widetilde{Z}}{\partial p_{z}}+\frac{\partial \widetilde{Y}}{\partial p_{z}}\right)\right]
\end{aligned}
$$

which gives the "standard" Ramsey-like system:

$$
\begin{aligned}
\frac{p_{x}-\left(c_{1}+c_{2}\right)}{p_{x}} & =\frac{\lambda}{1+\lambda} \frac{1}{\varepsilon_{X}}+\left[p_{z}-c_{2}\right]\left(\frac{\left(\partial Z / \partial p_{x}\right)}{\left(-\partial X / \partial p_{x}\right)}+\frac{\left(\partial Y / \partial p_{x}\right)}{\left(-\partial X / \partial p_{x}\right)}\right) \\
{\left[\frac{p_{z}-c_{2}}{p_{z}}\right] } & =\frac{\lambda}{(1+\lambda)} \frac{1}{\sigma_{Y+Z}}(Z+Y)+\left[p_{x}-\left(c_{1}+c_{2}\right)\right] \frac{\partial \widetilde{X} / \partial p_{z}}{\left(-\partial \widetilde{Z} / \partial p_{z}\right)-\partial \widetilde{Y} / \partial p_{z}}
\end{aligned}
$$

where

$\varepsilon_{X}=\frac{p_{x}}{X}\left(\frac{-\partial X}{\partial p_{x}}\right) \quad$ and $\quad \sigma_{Y+Z}=\frac{p_{z}}{Z+Y}\left(\frac{-\partial \widetilde{Z}}{\partial p_{z}}+\frac{-\partial \tilde{Y}}{\partial p_{z}}\right)=\frac{Z}{Z+Y} \sigma_{Z}+\frac{Y}{Z+Y} \sigma_{Y}$,

and

In matrix notation the FOC write:

$$
\sigma_{Z}=\frac{p_{z}}{Z}\left(\frac{-\partial \widetilde{Z}}{\partial p_{z}}\right) \quad \text { and } \quad \sigma_{Y}=\frac{p_{z}}{Y}\left(\frac{-\partial \widetilde{Y}}{\partial p_{z}}\right) .
$$

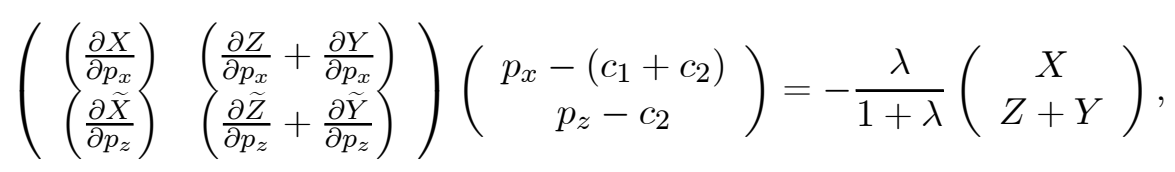

which is easily be solved to yield successively

$$
\begin{aligned}
p_{x}-\left(c_{1}+c_{2}\right) & =-\frac{\lambda}{1+\lambda} \frac{X\left(\frac{\partial \widetilde{Z}}{\partial p_{z}}+\frac{\partial \widetilde{Y}}{\partial p_{z}}\right)-(Z+Y)\left(\frac{\partial Z}{\partial p_{x}}+\frac{\partial Y}{\partial p_{x}}\right)}{\left(\frac{\partial X}{\partial p_{x}}\right)\left(\frac{\partial \widetilde{Z}}{\partial p_{z}}+\frac{\partial \widetilde{Y}}{\partial p_{z}}\right)-\left(\frac{\partial \widetilde{X}}{\partial p_{z}}\right)\left(\frac{\partial Z}{\partial p_{x}}+\frac{\partial Y}{\partial p_{x}}\right)}, \\
p_{z}-c_{2} & =-\frac{\lambda}{1+\lambda} \frac{\left(\frac{\partial X}{\partial p_{x}}\right)(Z+Y)-\left(\frac{\partial \widetilde{X}}{\partial p_{z}}\right) X}{\left(\frac{\partial X}{\partial p_{x}}\right)\left(\frac{\partial \widetilde{Z}}{\partial p_{z}}+\frac{\partial \widetilde{Y}}{\partial p_{z}}\right)-\left(\frac{\partial \widetilde{X}}{\partial p_{z}}\right)\left(\frac{\partial Z}{\partial p_{x}}+\frac{\partial Y}{\partial p_{x}}\right)},
\end{aligned}
$$


and

$$
\begin{aligned}
\frac{p_{x}-\left(c_{1}+c_{2}\right)}{p_{x}} & =\frac{\lambda}{1+\lambda} \frac{\frac{p_{z}}{Z+Y}\left(\frac{-\partial \widetilde{Z}}{\partial p_{z}}+\frac{-\partial \widetilde{Y}}{\partial p_{z}}\right)+\frac{p_{z}(Z+Y)}{p_{x} X}\left(\frac{p_{x}}{Z+Y}\right)\left(\frac{\partial Z}{\partial p_{x}}+\frac{\partial Y}{\partial p_{x}}\right)\left(\frac{-\partial X}{\partial p_{x}}\right) \frac{p_{z}}{Z+Y}\left(\frac{-\partial \widetilde{Z}}{\partial p_{z}}+\frac{-\partial \widetilde{Y}}{\partial p_{z}}\right)-\left(\frac{p_{z}}{X}\right)\left(\frac{\partial \widetilde{X}}{\partial p_{z}}\right)\left(\frac{p_{x}}{Z+Y}\right)\left(\frac{\partial Z}{\partial p_{x}}+\frac{\partial Y}{\partial p_{x}}\right)}{} \\
& =\frac{\lambda}{1+\lambda} \frac{\sigma_{Y+Z}+\frac{p_{z}(Z+Y)}{p_{x} X} \sigma_{(Z+Y) X}}{\varepsilon_{X} \sigma_{Y+Z}-\sigma_{X Z} \sigma_{(Z+Y) X}} \\
\frac{p_{z}-c_{2}}{p_{z}} & =\frac{\lambda}{1+\lambda} \frac{\frac{p_{x}}{X}\left(\frac{-\partial X}{\partial p_{x}}\right)+\frac{p_{x} X}{p_{z}(Z+Y)}\left(\frac{p_{z}}{X}\right)\left(\frac{\partial \widetilde{X}}{\partial p_{z}}\right)}{\left(\frac{p_{x}}{X}\right)\left(\frac{\partial X}{\partial p_{x}}\right)\left(\frac{p_{z}}{Z+Y}\right)\left(\frac{\partial \widetilde{Z}}{\partial p_{z}}+\frac{\partial \widetilde{Y}}{\partial p_{z}}\right)-\left(\frac{p_{z}}{X}\right)\left(\frac{\partial \widetilde{X}}{\partial p_{z}}\right)\left(\frac{p_{x}}{Z+Y}\right)\left(\frac{\partial Z}{\partial p_{x}}+\frac{\partial Y}{\partial p_{x}}\right)} \\
& =\frac{\lambda}{1+\lambda} \frac{\varepsilon_{X}+\frac{p_{x} X}{p_{z}(Z+Y)} \sigma_{X Z}}{\varepsilon_{X} \sigma_{Y+Z}-\sigma_{X Z} \sigma_{(Z+Y) X}}
\end{aligned}
$$

where

$$
\begin{aligned}
\sigma_{X Z} & =\left(\frac{p_{z}}{X}\right)\left(\frac{\partial \tilde{X}}{\partial p_{z}}\right) \\
\sigma_{(Z+Y) X} & =\left(\frac{p_{x}}{Z+Y}\right)\left(\frac{\partial Z}{\partial p_{x}}+\frac{\partial Y}{\partial p_{x}}\right) \\
& =\left(\frac{Z}{Z+Y}\right)\left(\frac{p_{x}}{Z} \frac{\partial Z}{\partial p_{x}}\right)+\left(\frac{Y}{Z+Y}\right)\left(\frac{p_{x}}{Y} \frac{\partial Y}{\partial p_{x}}\right) \\
& =\left(\frac{Z}{Z+Y}\right) \sigma_{Z X}+\left(\frac{Y}{Z+Y}\right) \sigma_{Y X},
\end{aligned}
$$

Rearranging these expressions and defining superelasticities in the usual way:

$$
\begin{aligned}
\widehat{\sigma}_{X} & =\frac{\varepsilon_{X} \sigma_{Y+Z}-\sigma_{X Z} \sigma_{(Z+Y) X}}{\sigma_{Y+Z}+\sigma_{X Z}} \\
\widehat{\sigma}_{Y+Z} & =\frac{\sigma_{Y+Z} \varepsilon_{X}-\sigma_{X Z} \sigma_{(Z+Y) X}}{\varepsilon_{X}+\sigma_{(Z+Y) X}} .
\end{aligned}
$$

yields expression (19) and (20). To simplify the expression we have used the property that demand functions for $\widetilde{X}$ and for $(\widetilde{Z}+\widetilde{Y})$ have symmetric cross derivatives:

$$
\begin{aligned}
\frac{\partial \widetilde{X}}{\partial p_{z}} & =\frac{\partial X}{\partial p_{z}}+\frac{\partial X}{\partial p_{y}} \\
& =x_{f}\left(p_{x}, p_{y}, \widetilde{k}\right) g(\widetilde{k})+\int_{p_{x}-p_{z}}^{\bar{k}} \frac{\partial x_{f}\left(p_{x}, p_{y}, k\right)}{\partial p_{y}} g(k) d k \\
& =z\left(p_{z}+\widetilde{k}+, p_{y}, \widetilde{k}\right) g(\widetilde{k})+\int_{p_{x}-p_{z}}^{\bar{k}} \frac{\partial y\left(p_{x}, p_{y}, k\right)}{\partial p_{x}} g(k) d k \\
& =\frac{\partial Z}{\partial p_{x}}+\frac{\partial Y}{\partial p_{x}}=\frac{\partial \widetilde{Z}}{\partial p_{x}}+\frac{\partial \widetilde{Y}}{\partial p_{x}}
\end{aligned}
$$


which in turn arises because.

$$
\frac{\partial x_{f}\left(p_{x}, p_{y}, k\right)}{\partial p_{y}}=\frac{\partial y\left(p_{x}, p_{y}, k\right)}{\partial p_{x}}
$$

and which implies $p_{z}(Z+Y) \sigma_{(Z+Y) X}=p_{x} X \sigma_{X Z}$.

\section{B Derivation of expressions (22) and (23)}

First observe that in this case we have:

$$
\begin{aligned}
& \frac{\partial \widetilde{X}}{\partial p_{x}}=\frac{\partial x_{h}}{\partial p_{x}} \text { and } \frac{\partial \widetilde{X}}{\partial p_{z}}=0 \\
& \frac{\partial \widetilde{Y}}{\partial p_{x}}=0 \text { and } \quad \frac{\partial \widetilde{Y}}{\partial p_{z}}=\frac{\partial Y}{\partial p_{y}} \\
& \frac{\partial \widetilde{Z}}{\partial p_{x}}=0 \text { and } \quad \frac{\partial \widetilde{Z}}{\partial p_{z}}=\frac{\partial Z}{\partial p_{z}}
\end{aligned}
$$

Using these properties to simplify the FOCs (27) and (28) one obtains

$$
\begin{aligned}
& \frac{\partial L}{\partial p_{x}}=\lambda x_{h}+(1+\lambda)\left[p_{x}-\left(c_{1}+c_{2}\right)\right] \frac{\partial x_{h}}{\partial p_{x}} \\
& \frac{\partial L}{\partial p_{z}}=\lambda(Z+Y)+(1+\lambda)\left[p_{z}-c_{2}\right]\left(\frac{\partial Z}{\partial p_{z}}+\frac{\partial Y}{\partial p_{y}}\right),
\end{aligned}
$$

which can easily be rearranged to yield (22) and (23). 